\title{
Well Differentiated Neuroendocrine Uncommon Primary Breast Carcinoma: A Case Report
}

\section{Angela Strazzanti, $M D^{1^{*}}$, Claudio Trovato, $M D^{1}$, Angelo Caponnetto, $M D^{1}$, Santi Gangi, $M D^{1}$, Loredana $R$ Villari, $M D^{2}$, Pietro Gangemi, $M D^{2}$ and Francesco Basile, $M D^{1}$}

${ }^{1}$ Scuola di Facoltà di Medicina, General Surgery Catania, Universita degli Studi di Catania, Italy

${ }^{2}$ U.O.C. di Anatomia Patologica e Biologia Molecolare, Azienda Ospedaliera Policlinico-Vittorio Emanuele Catania, Italy

*Corresponding author: Angela Strazzanti, MD, Scuola di Facoltà di Medicina, General Surgery Catania, Universita degli Studi di Catania, 95124, Sicily, Italy, Tel: +393925743156

\begin{abstract}
Neuroendocrine breast carcinomas (NEBC) are rarely malignant with a frequency of less than $0.1-0.3 \%$ of all breast tumors. The actual incidence of NEBC in BC (Breast Cancer) populations being still largely unknown due to the lack of a clear cut diagnostic criteria. In 2003, the World Health Organization (WHO) Classification of Tumors of the Breast and Female Genital Organs definitely established that the immunohistochemical expression of NE markers is the unique requirement for NEBC diagnosis [1] in more than $50 \%$ of the tumor cell population. In the $2012 \mathrm{WHO}$ Classification of the Tumors of the Breast these entities were collected in another chapter, among the special subtypes: Carcinomas with neuroendocrine features, which encompass the categories of Neuroendocrine tumors which are well differentiated, Neuroendocrine carcinoma which is a poorly differentiated/small cell carcinoma and Invasive breast carcinoma with neuroendocrine differentiation.

We would like to report the case of a 67-year-old woman with a rare neuroendocrine well differentiated breast cancer detected accidentally during a PET performed as a follow up in the treatment of a bone plasmacitoma. The result of histological examination was well differentiated primary neuroendocrine tumor of the breast with CKPan+, SYn+; CK7- and CK5-; ER+, Pr+, HER2- and ki67 < 5\% [1].

The prognosis of NECB is not different from other invasive breast carcinomas and the most important prognostic factor is the tumor grade $(G)$. However, there is no standard treatment and patients should be treated similarly to patients with invasive ductal carcinoma, NOS (Not Otherwise Specified), the choice of therapy depending on the size of the tumor, the degree of differentiation, the clinical stage, and the hormonal status.
\end{abstract}

\author{
Abbreviations \\ NEBC: Neuroendocrine Breast Carcinoma; BC: Breast \\ Cancer; NOS: Not Otherwise Specified
}

\section{Introduction}

Neuroendocrine carcinomas are rarely malignant tumors which are mostly located in the lungs and the gastrointestinal tract. Primary location of such tumors in the breast is extremely rare, corresponding to less than $0.1-0.3 \%$ of all breast tumors and less than $1-3 \%$ of all neuroendocrine tumors. In fact, the true incidence of the disease is difficult to assess, because the immunohistochemistry neuroendocrine markers are not routinely used in breast tumors [2].

NECB was originally reported by Feyrter and Hartmann [3] in 1963 as an invasive breast cancer morphologically similar to intestinal carcinoids. In 1977, Cubilla and Woodruff [4] described eight other cases of breast cancer with a carcinoid growth pattern, providing for the first time histopathological classification together with a clinical and prognostic analysis of NECB.

Focal neuroendocrine differentiation can be found in different histological types of breast carcinoma including in situ and invasive ductal or invasive lobular ones. Up to $30 \%$ of invasive carcinomas and particularly mucinous carcinomas may be detected by means of histochemical and immunohistochemical analysis.

Citation: Strazzanti A, Trovato C, Caponnetto A, Gangi S, Villari LR, et al. (2019) Well Differentiated Neuroendocrine Uncommon Primary Breast Carcinoma: A Case Report. Int J Cancer Clin Res 6:108. doi. org/10.23937/2378-3419/1410108

Accepted: April 09, 2019; Published: April 11, 2019

Copyright: (C) 2019 Strazzanti A, et al. This is an open-access article distributed under the terms of the Creative Commons Attribution License, which permits unrestricted use, distribution, and reproduction in any medium, provided the original author and source are credited. 
According to $\mathrm{WHO}$, the neuroendocrine carcinoma is a tumor with positive immunoreactivity to neuroendocrine markers, synaptophysin and/or chromogranin, in at least $50 \%$ of tumor cells [5].

In 2012, WHO classified these tumors into three categories:

(1) Well differentiated neuroendocrine tumor;

(2) Poorly differentiated/small cell carcinoma. The most commontumor, which is CK7+ and Ck20, is morfologically similar to the small lung cell carcinoma which is negative for both markers;

3) Invasive breast carcinoma with neuroendocrine differentiation $\mathrm{Er}+, \mathrm{PR}+[6]$.
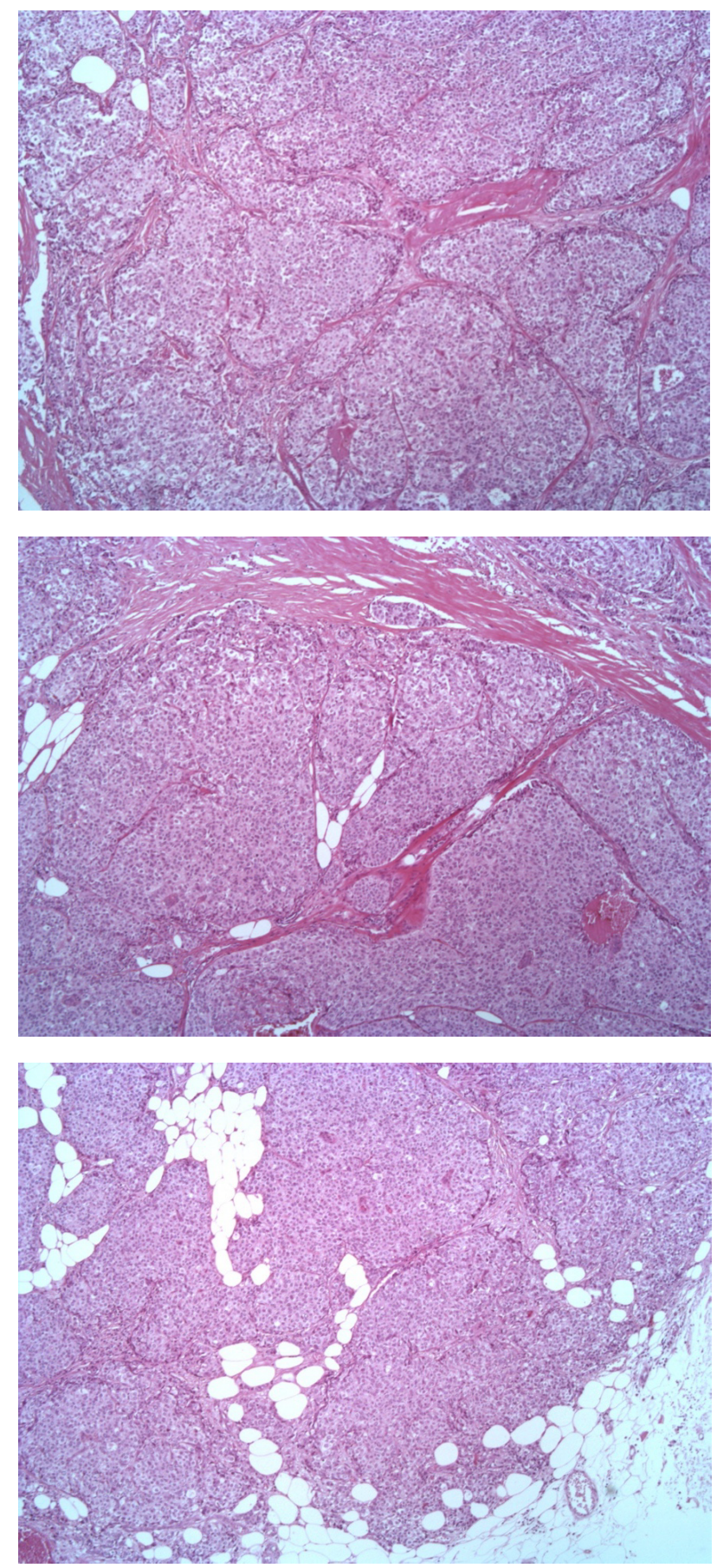

Figure 1: EE ingrandimento 5X.
Neuroendocrine differentiation is an independent adverse prognostic factor for the first two specific categories and the overall survival. The average of onset is 64-years-old [7].

Sapino, et al. describe 5 histological subtypes: Solid, alveolar, small cell, solid papillary and mucinous [8].

Adjuvant treatment should follow the same medical plan as the other types of invasive breast cancer. An accurate diagnosis of NECB is also important in the metastatic setting, in which a multimodality approach including specific therapies such as peptide receptor and radionuclide therapy can be considered [20].

\section{Case Presentation}

We are here describing the case of a 67-yearold woman with a well-differentiated plasmocellular plasmacytoma which was diagnosed in 2011; with restriction k, FISH positive for del17p13.

Because of that she underwent chemotherapy according to the VTD scheme (Velcade, Thalidomide, Dexamethasone) + zometa, with complete response to the treatment.

In 2018 the patient complained of widespread osteoarticular pains for which a BOM was carried out, which was negative. After that, a CT total body showed a 16-mm diameter swelling in the upper inner quadrant of the left breast. She also underwent a PET 18-FDG that
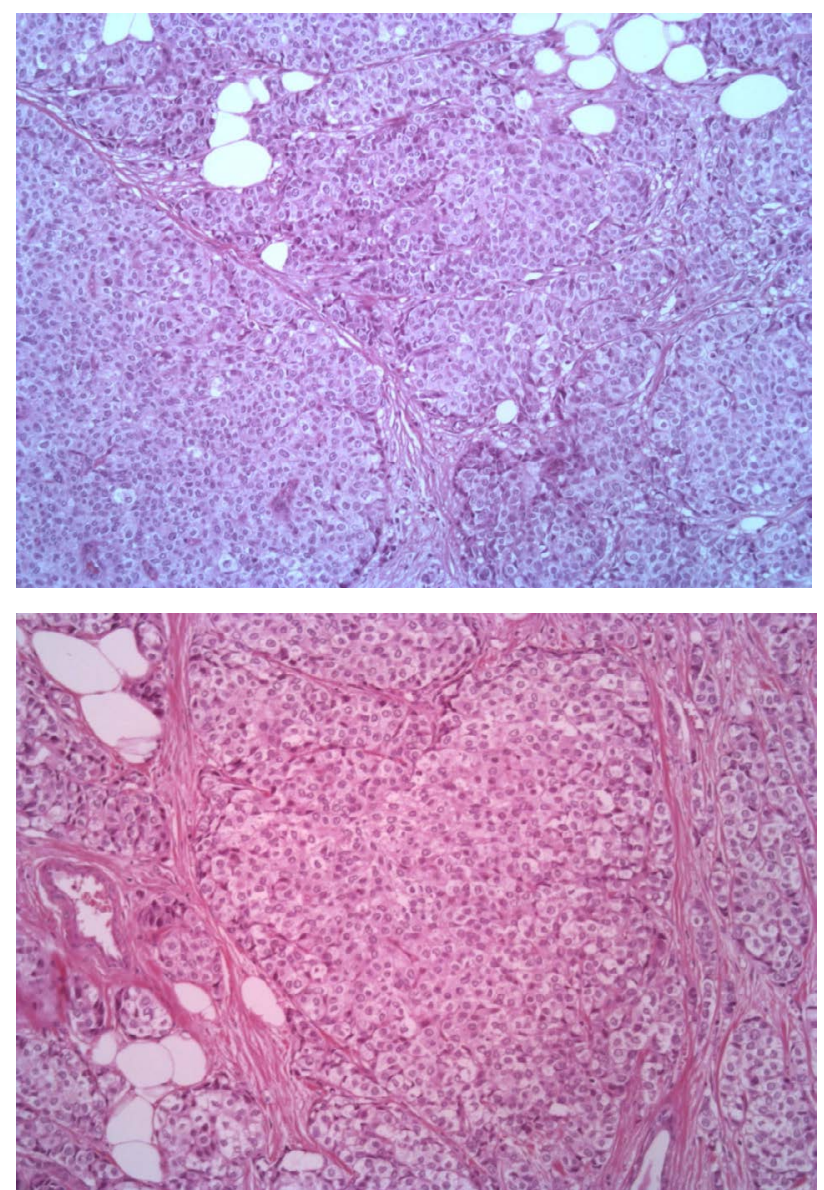

Figure 2: EE 10X. 
showed an increase of hyper metabolism of 18 FDG localized in the left breast and also in the left iliac branch. For this reason a PET Ga DOTATOC was performed to exclude a plasmacitoma origin or a primary lesion. The PET confirmed aniliac lesion in relation to a plasmacitoma. A tru-cut biopsy instead showed a ductal carcinoma in the left breast. A quadrantectomy and sentinel limpho node biopsy were performed. The histological result was a well differentiated primary neuroendocrine cancer of the breast: pT1c N0, G1, $1.7 \mathrm{~cm}$ with CKPan+, SYn+; CK7- and CK5-; ER 90\%, Pr 80\%, Herb negative and ki67 $<5 \%$. The sentinel node biopsy was negative. Since according to literature there is no standard treatment patients should be treated similarly to patients with ductal invasive carcinoma. Consequently, she underwent radiotheraphy and hormonal therapy.
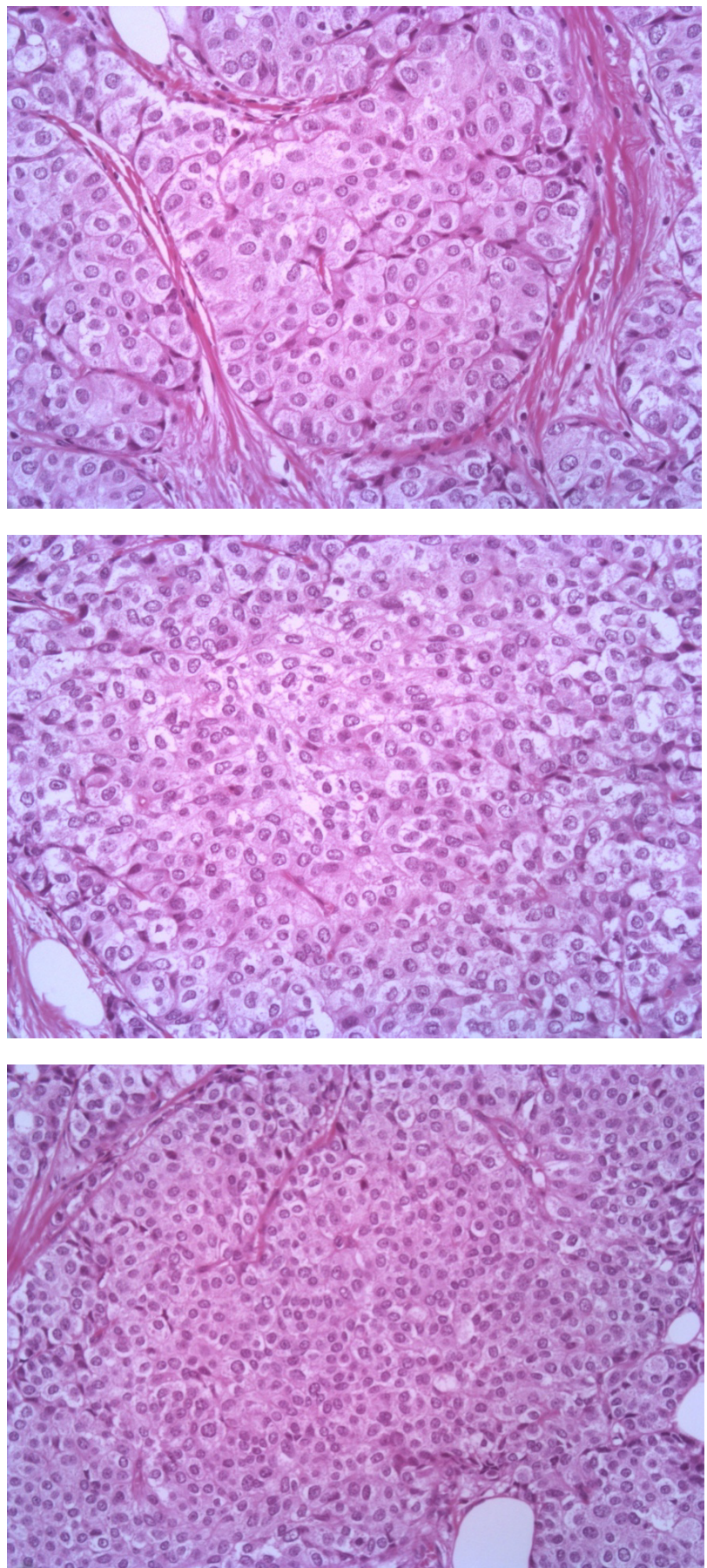

Figure 3: EE 20X.

\section{Discussion}

The histogenesis of NECB has not been clearly defined yet. One of the theories suggests the development of NECB from neuroendocrine cells which are constitutively present in the breast, but these cells have not been consistently found in normal breast tissue [9]. Furthermore, neuroendocrine cell hyperplasia or in situ neuroendocrine carcinoma that would support a progression from noninvasive to invasive neuroendocrine tumors have been rarely reported [10]. According to another theory, NECB would instead result from an early divergent differentiation of breast cancer stem cells into both neuroendocrine and epithelial lines [11]. This hypothesis is consistent with the observation that NECB is always a mixed tumor including endocrine and exocrine cells, and it is also consistent with molecular studies showing that in NECB the neuroendocrine cells are clonally related to the intraductal component of the tumor [12].

NECB is often positive for hormone receptors, whereas HER2 is almost always negative. Accordingly, the gene expression profile analysis has revealed that NECB belongs to the Luminal A subtype [13]. A luminal B phenotype, defined by immunohistochemistry as hormone receptor-positive tumor with high proliferation

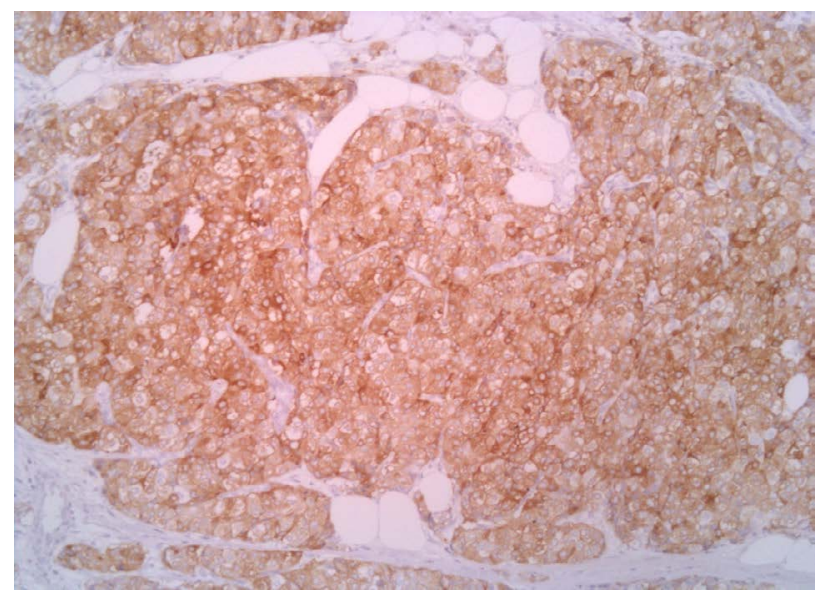

Figure 4: Synaptophisyn 10X.

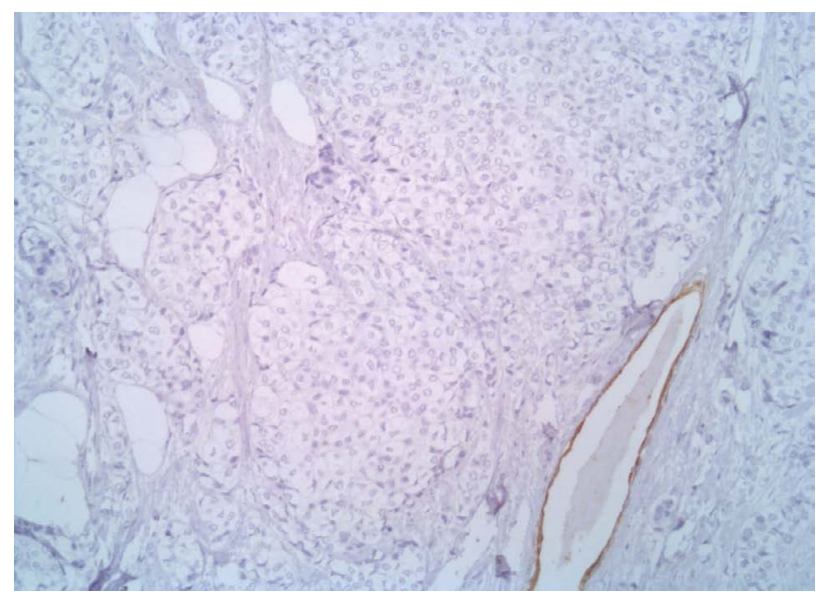

Figure 5: CK7 10X. 
index (i.e., Ki67 > 14\%), accounts for approximately $50 \%$ of NECB [14]. However, there are also reports of HER2-positive NECB [15] and small-cell carcinoma of the breast with basal-like characteristics.

The tumor consists of densely cellular, solid nests (prevalent aspect in our case) and trabeculae of round cells of small-medium size, with abundant clear cytoplasm, separated by delicate fibrovascular stroma. The cells have round, regular nuclei and show some palizading arrangement at the periphery of the nests.



Figure 6: Synaptophisyn 20X.

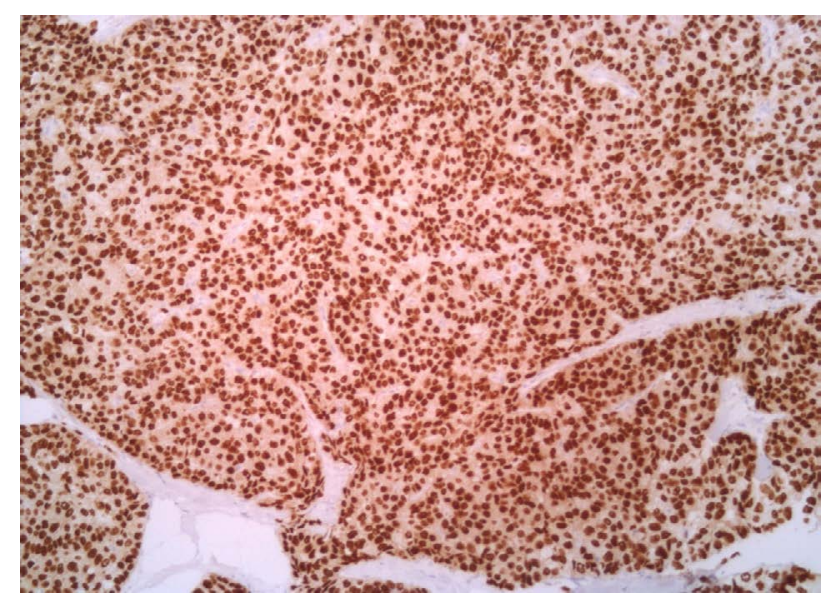

Figure 7: Estrogenic receptor 10X.

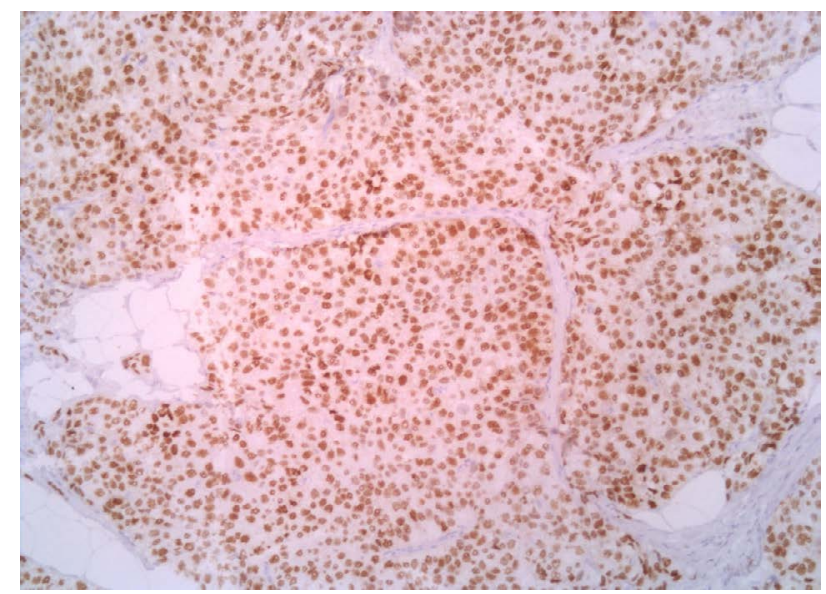

Figure 8: Progestinic receptor 10X.
The immunophenotype was coherent with the diagnosis, showing bright positivity for synaptophysin, while CK5 and CK7 were negative.

Estrogenic and progestinic receptors showed diffuse positivity as well, with low proliferative fraction (Ki67/ Mib1 < 5\%) and no amplification of HER2 gene (data not shown) (Figure 1, Figure 2, Figure 3, Figure 4, Figure 5, Figure 6, Figure 7, Figure 8, Figure 9 and Figure 10).

Focal neuroendocrine differentiation could be observed in various histological subtypes of mammary carcinoma [21], including in situ carcinoma and invasive ductal, lobular, colloid, or papillary carcinoma. Primary neuroendocrine cancer of the breast must be distinguished from a metastatic lesion from other sites. Clinical features and morphology are not helpful to distinguish NECB from other subtypes of breast cancer; therefore, immunohistochemistry markers for neuroendocrine differentiation, mainly chromogranin and synaptophysin, should be routinely used to confirm the diagnosis, especially in the cases of mucinous or solid papillary carcinoma in which the suspicion of NECB is high. It is also useful to verify the absence of clinical evidence of a concurrent primary neuroendocrine carcinoma elsewhere. Most published studies about neuroendocrine breast carcinoma emphasize anatomopatho-

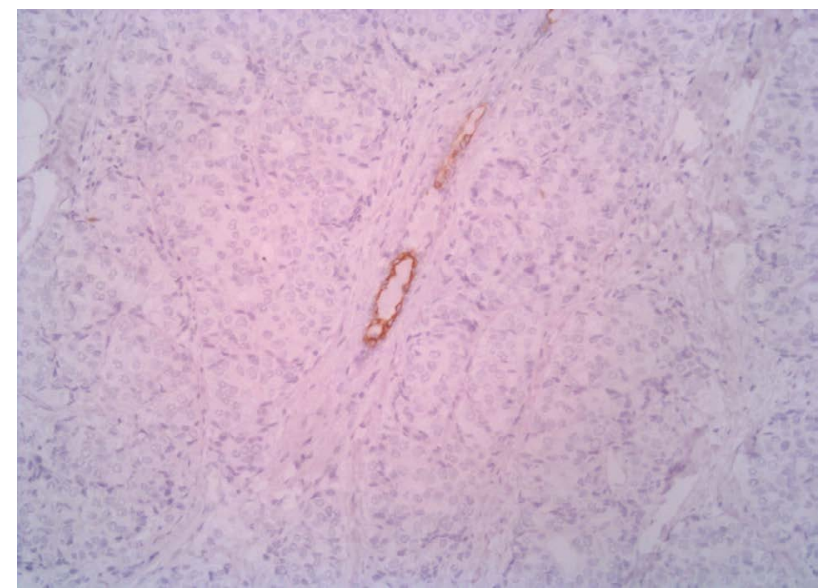

Figure 9: CK5 10X (with positive control - normal duct entrapped in the neoplasm).

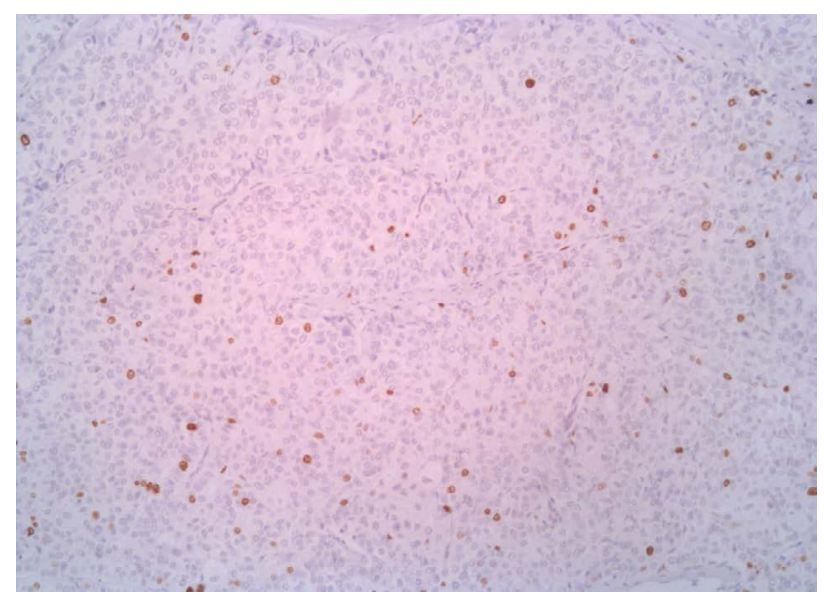

Figure 10: Ki67 10X. 
logical findings, with little reference to imaging results [1618].

The presence of an in situ ductal carcinoma is a histological evidence that breast is the primary organ of origin [19].

\section{Conclusions}

A review of the literature, which rarely records welldifferentiated neuroendocrine breast carcinoma, let us conclude that the NECB evolves similarly to the invasive ductal carcinoma and therefore the prognosis depends on the classical criteria of the TNM. The treatment plan may be the same as that of the invasive ductal breast carcinoma, as NECB may later show liver and bone secondarily metastases.

Up to now, our patient is in good general conditions. We have not detected any recurrences of locoregional disease, notwithstanding the presence of bone lesions from plasmacytoma under control by means of specific therapy. Axillary lymph nodes are being tested by ultrasound scan every three months and have not showed pathological changes so far. In conclusion, a well-differentiated neuroendocrine carcinoma is supposed to have a consistent follow-up. It would be useful to perform immunohistochemistry markers even in cases of invasive ductal tumors with in situ component and/or with mucinous component.

\section{Conflict of Interest}

There is no conflict of interest with any of the authors involved in this paper.

\section{Funding Source}

None.

\section{Ethical Approval}

No ethics approval was needed.

\section{Author contributions}

Trovato Agata: Reviewing and editing the article.

\section{Consent}

Written informed consent was obtained from the patient for publication of this case report and accompanying images. A copy of the written consent is available for review by the Editor-in-Chief of this journal on request.

\section{References}

1. Lena Marinova, Doroteya Malinova, Snezhinka Vicheva (2016) Primary Neuroendocrine Carcinoma of the Breast: Histopathological Criteria, Prognostic Factors, and Review of the Literature.

2. Lakhani SR, Ellis IO, Schnitt SJ (2012) World Health Organization Classification of Tumours of the Breast. $\left(4^{\text {th }}\right.$ edn), IARC Press, Lyon, France.

3. Feyrter F, Hartmann G (1963) On the carcinoid growth form of the carcinoma mammae, especially the carcinoma solidum (gelatinosum) mammae. Frankf Z Pathol 73: 24-39.
4. Cubilla AL, Woodruff JM (1997) Primary carcinoid tumor of the breast: A report of eight patients. Am Surg Pathol 1: 283-292.

5. Tavassoli FA, Devilee P (2003) Pathology and Genetics: Tumours of the Breast and Female Genital Organs. ( $3^{\text {rd }}$ edn), IARC Press, Lyon, France.

6. Bussolati G, Badve S (2012) Carcinomas with neuroendocrine features. In: Lakhani SR, Ellis IO, Schnitt SJ, Tan PH, van de Vijver MJ, WHO Classification of Tumours of the Breast. IARC Press, Lyon, France 62-63.

7. Wang J, Wei B, Albarracin CT, Hu J, Abraham SC, et al. (2014) Invasive neuroendocrine carcinoma of the breast: a population-based study from the surveillance, epidemiology and end results (SEER) database. BMC Cancer 14: 147.

8. Sapino A, Righi L, Cassoni P, Papotti M, Pietribiasi F, et al. (2000) Expression of the neuroendocrine phenotype in carcinomas of the breast. Seminars in Diagnostic Pathology 17: $127-137$

9. Bussolati G, Gugliotta P, Sapino A, Eusebi V, Lloyd RV (1985) Chromogranin-reactive endocrine cells in argyrophilic carcinomas ("carcinoids") and normal tissue of the breast. Am J Pathol 120: 186-192.

10. Miura K, Nasu H, Ogura H (2012) Double neuroendocrine ductal carcinomas in situ coexisting with a background of diffuse idiopathic neuroendocrine cell hyperplasia of breast: A case report and hypothesis of neuroendocrine tumor development. Pathol Int 62: 331-334.

11. Miremadi A, Pinder SE, Lee AH, Bell JA, Paish EC, et al. (2002) Neuroendocrine differentiation and prognosis in breast adenocarcinoma. Histopathology 40: 215-222.

12. Hoang MP, Maitra A, Gazdar AF, Albores-Saavedra J (2001) Primary mammary small-cell carcinoma: A molecular analysis of 2 cases. Hum Pathol 32: 753-757.

13. Weigelt B, Horlings HM, Kreike B, Hayes MM, Hauptmann $M$, et al. (2008) Refinement of breast cancer classification by molecular characterization of histological special types. J Pathol 216: 141-150.

14. Bogina G, Munari E, Brunelli M, Bortesi L, Marconi M, et al. (2015) Neuroendocrine differentiation in breast carcinoma: Clinicopathological features and outcome. Histopathology 68: 422-432.

15. Fujimoto $Y$, Yagyu R, Murase $K$, Kawajiri $H$, Ohtani $H$, et al. (2007) A case of solid neuroendocrine carcinoma of the breast in a 40-year-old woman. Breast Cancer 14: 250-253.

16. Kinoshita S, Hirano A, Komine K (2008) Primary small-cell neuroendocrine carcinoma of the breast: report of a case. Surg Today 38: 734-738.

17. López-Bonet E, Alonso-Ruano M, Barraza G, VazquezMartin A, Bernadó L, et al. (2008) Solid neuroendocrine breast carcinomas: incidence, clinico-pathological features and immunohistochemical profiling. Oncol Rep 20: 1369-1374.

18. Ooi A, Ohta T, Mai M, Naknishi I, Takahasi Y (1988) Primary breast carcinoma with extensive endocrine differentiation: an immunohistochemical and immunoelectron microscopic study. Surg Pathol 11: 277-284.

19. Tajima S, Horiuchi H (2013) Neuroendocrine tumor, well differentiated, of the breast: a relatively high-grade case in the histological subtype. Case Reports in Pathology.

20. Strazzanti A, Trovato C, S Gangi, F Basile (2018) Breast tuberculosis cases rising in Sicily. Int J Surg Case Rep 53: 9-12.

21. Strazzanti A, Trovato C, S Gangi, N Pacini, F Basile (2018) Contralateral lymph node metastasis in a woman with new primary breast cancer: Systemic desease or locoregional diffusion? Int J Surg Case Rep 53: 400-402. 